\title{
Structural Stability Analysis of Steel Tubular Scaffold with Couplers Based on Direct Analysis Method
}

\author{
Junce ZHANG, Fankui ZENG*, Huan MA
}

\begin{abstract}
Direct analysis method (DAM) is a second-order nonlinear analysis approach widely used for its high accuracy and simple flow. It is suitable for pinpointing the internal force response and nonlinear behavior of steel tubular scaffold with couplers (STSC), which has large initial defects arising from repeated use. This paper firstly quantifies the initial defects of steel tubes of the scaffold and draws the column curves of the tubes with different initial bending and residual stresses. On this basis, a reference value was derived for the overall initial defect of the tubes with different initial bending. Through a calculation example, a finite-element model was established in view of the overall initial defect, the initial defects of components, and the nonlinear semi-stiffness of nodes and the DAM flow was introduced for the STSC. The calculation example verifies the rationality of the reference value for the overall initial defect, making it easier to apply the DAM to actual engineering.
\end{abstract}

Keywords: Direct Analysis Method (DAM); Initial Defects; Nonlinear Analysis; Steel Tubular Scaffold with Couplers (STSC); Ultimate Bearing Capacity

\section{INTRODUCTION}

Steel tubular scaffold with couplers (STSC) is a temporary support structure. It is susceptible to collapse in the case of insufficient stiffness, stability and bearing capacity, which will undermine the safety of the entire construction process. To prevent the collapse, it is important to step up the work safety management of the scaffold. More importantly, it is a must to fully consider the nonlinear behavior of the scaffold and realistically reflect the structural response in the design phase.

Direct analysis method (DAM) is the second-order nonlinear analysis approach. Chen et al. [1] were the first to propose the concept of advanced analysis: in order to predict the carrying capacity and instability mode of a structure, it is critical to consider all the factors that might affect the result stability and overall carrying capacity and allow the rod pieces to undergo plastic development and redistribution of internal force. Taking single-layer reticulated shell as an example, Wang et al. [2] explained the application of DAM in spatial structureand elaborated on the feasibility and superiority of the method. For the STSC with obvious second-order effect and clearly nonlinear rod pieces, DAM-based calculation and analysis can reflect the real structural response. Liu et al. [3] summarized the direct analysis steps and bearing capacity formula for DAM application, and evaluated the research status of ensuring the overall stability of components with DAM. Yu et al. [4] used DAM to check the hydrochloric acid and cross section of the cable structure with the stress ratio of 1.1, and confirmed that DAM guarantees the stability of the structure, and saves steel compared with the calculated length method. Zhou [5] designed the steel roof of the tennis hall at Yanghang Sports Center with DAM, and obtained accurate load correspondence and component parameters. During the DAM analysis, the design flow should be optimized in light of the second-order effect, and the influence of node semi-stiffness on internal force distribution.

To analyze the STSC with DAM, the following three factors must be determined: the overall initial defect of the structure, the initial defects of components and the nonlinear semi-stiffness of nodes. On the first two factors,
$\mathrm{Hu}$ and Zeng [6] simulated the generalized initial defects by applying an imaginary horizontal force of $1.2 \%-2.5 \%$ of the ultimate bearing capacity and achieved finiteelement results fit in with test results. Lu et al. [7] carried out prototype tests and finite-element analysis on 15 highdensity scaffolds, described initial defects in the form of the lowest order mode of eigenvalue buckling analysis and applied them proportionally to the model; but the specific proportion was not mentioned. Yuan et al. [8] carried out nonlinear buckling analysis on the stable bearing capacity of the STSC, added a horizontal force about $1 \%$ of vertical axial pressure at the junction between horizontal and vertical rods during the finite-element analysis and observed significant influence of initial defects and node semi-stiffness on stable bearing capacity. Yao [9] studied the calculation method of the initial defects of the STSC, and compared the impacts of different defect simulation approaches on stable bearing capacity. Based on steel structure theory, Liu and Shu [10] derived the overall initial defect of compressive components with different crosssections, and verified the theoretical results on analysis software. For node semi-stiffness, Zhang et al. [11] proposed a calculation model of semi-stiff nodes based on the results of full scaffold test, and inverted the node stiffness: $25 \mathrm{kN} \cdot \mathrm{m} / \mathrm{rad}$. Prabhakaran et al. [12] presented the second-order algorithm for spatial scaffolds, which considers the nonlinear behaviors of scaffolds with semistiff connections. However, the bending angle of each node was not taken into account, due to the lack of test data. Hu et al. [13] simulated the semi-stiffness of nodes with the rotating spring, and calculated the rotational stiffness of nodes $(85.96 \mathrm{kN} \cdot \mathrm{m} / \mathrm{rad})$ at the tightening torque of $40 \mathrm{~N} \cdot \mathrm{m}$. Many other scholars have explored the semi-stiffness of nodes. Nevertheless, they simplified node semi-stiffness during the simulation and considered the rotational stiffness as linear. In fact, node semi-stiffness is strongly nonlinear. Zou et al. [14] adopted a trilinear node model to simulate the node semi-stiffness of bow buckle scaffold and considered the nonlinearity of the initial geometry of components, thereby realizing a high simulation accuracy.

In summary, the DAM analysis of STSC requires a highly refined modeling process. The model must consider the initial defects of the structure and components, as well 
as the semi-stiffness of nodes. But these factors are simplified by most scholars during the simulation. Since the stable carrying capacity of the defect-sensitive STSC depends greatly on the size of initial defects, this paper tries to accurately quantify the overall initial defect, the initial defects of components and nonlinear semi-stiffness of nodes, such that the DAM can be applied to actual engineering more effectively.

\section{INITIAL DEFECTS OF COMPONENTS}

Due to repeated use of the STSC, the defects of the steel tubes will accumulate in each reuse. As a result, the STSC is highly sensitive to defects. According to the Technical Code for Safety of Steel Tubular Scaffold with Couplers in Construction (JGJ 130-2011) [15], the allowable deviation is defined as the initial bending of $3 \mathrm{~L} / 1,000$. During the repeated use, however, the initial geometric defects and material defects of the steel tubes keep increasing. It is not reasonable to adopt this value for design.

The cross-sectional features of the steel tubes of the scaffold obey the curve for Class b columns of Q235 steel. In the Standard for Design of Steel Structures (GB 500172017) [16], the initial bending is set to $L / 1,000$, and the residual stress is considered under specific modes. On that basis, this paper fits the column curves under different initial bending and residual stresses through ANSYS simulation. Considering the geometric and material defects of components, the obtained curves were used to fit the formula for stability coefficient of axial compression mentioned in GB 50017-2017. By the formula, the initial defects of components were inverted and a reference value was provided for the initial defects of STSC tubes under each initial bending. The quantified reference values help to improve the application of the DAM to actual STSCs.

Table 1 Geometric features of steel tubes

\begin{tabular}{|c|c|c|c|c|c|c|}
\hline $\begin{array}{l}\text { Outer diameter } D / \\
\mathrm{mm}\end{array}$ & $\begin{array}{l}\text { Wall thickness } t / \\
\text { mm }\end{array}$ & $\begin{array}{c}\text { Cross-sectional area } A \\
/ \mathrm{mm}^{2}\end{array}$ & $\begin{array}{l}\text { Inertial moment } I / \\
\mathrm{mm}^{4}\end{array}$ & $\begin{array}{c}\text { Cross-sectional modulus } \\
\qquad / \mathrm{mm}^{3}\end{array}$ & $\begin{array}{l}\text { Turning radius } I / \\
\text { mm }\end{array}$ & $\begin{array}{l}\text { Mass per meter } \\
\mathrm{kg} / \mathrm{m}\end{array}$ \\
\hline 48 & 3 & 424.12 & 107831.20 & 4492.97 & 15.95 & 3.33 \\
\hline
\end{tabular}

ANSYS simulation was performed to analyze the ultimate bearing capacity of steel tubes under different initial bending. Then, different calculation lengths and initial defects were written into the main program for batch calculation, revealing the relationship between stability coefficient and regularized slenderness ratio. On this basis, the column curves were plotted for different initial defects. During the simulation, the STSC steel tubes were configured as Q235 steel tubes with a yield strength of 235 $\mathrm{MPa}$, and a Poisson' ratio of 0.3 . The constitutive relationship of the steel pipes was simulated with bilinear kinematic hardening (BKIN) model; the nonlinear material was assumed to be ideally elastoplastic, and in line with the von Mises yield criterion. The geometric features of the steel tubes are listed in Tab. 1.

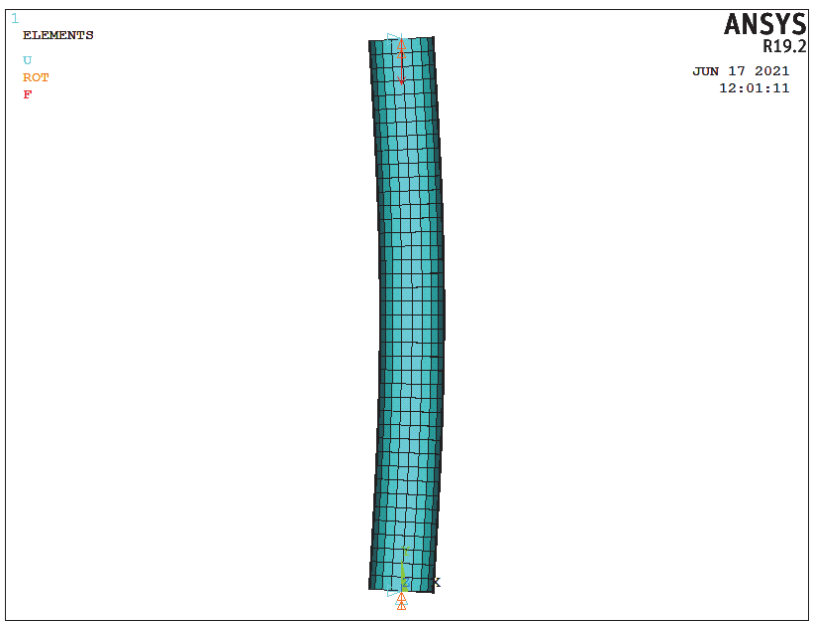

Figure 1 Steel tube model with initial bending of $L / 1,000$

For the ANSYS simulation, the steel tubes were simulated as BEAM188 elements. Both ends of each tube were hinged. An axial force $F$ was applied on the upper side until the steel tube witnessed the first plastic hinge. This is taken as the yield criterion. Then, the higher-order function was called and the arch length method was adopted for nonlinear analysis. Fig. 1 shows the established model, which considers the bending of L/1,000 (magnified by 20 times). Taking the above as the subprogram, different initial defects and slenderness ratios were set up in the main program to discuss the relationship between slenderness ratio $\lambda$ and stability coefficient $\varphi$. Finally, the curve between stability coefficient $\varphi$ and regularized slenderness ratio $\lambda_{n}$ was obtained.

The steel tubes are welded tubes with a Class b crosssection. As specified in GB 50017-2017, the $\varphi-\lambda_{n}$ of Class b cross-section can be calculated by [16]:

$$
\begin{aligned}
& \text { If } \lambda_{n} \leq 0.215, \\
\varphi= & 1-\alpha_{1} \lambda^{2} \\
& \text { If } \lambda_{n}>0.215, \\
\varphi= & \frac{1}{2 \lambda_{n}^{2}}\left[\left(a_{2}+a_{3} \lambda_{n}+\lambda_{n}^{2}\right)-\sqrt{\left(a_{2}+a_{3} \lambda_{n}+\lambda_{n}^{2}\right)^{2}-4 \lambda_{n}^{2}}\right]
\end{aligned}
$$

where, $a_{1}=0.65, a_{2}=0.965, a_{3}=0.300$.

By Euler's formula, the stability coefficient $\varphi$ can be calculated by:

$\varphi=\frac{1}{\lambda_{n}^{2}}$

Thus, the Euler solution, standard solution and ANSYS solution were obtained for the column curve of steel tubes under the initial bending of L/1,000 (Fig. 2).

As shown in Fig. 2, the ANSYS solution fell between the other two solutions, and slightly exceeded the standard solution. The difference between the ANSYS solution and regular solution is the influence of the residual stress of steel tubes on the stable carrying capacity of rod pieces. Taking the difference as the influence of steel tubes with a 
specific residual stress, the column curves under different initial bending and residual stresses can be obtained as Fig.

3.

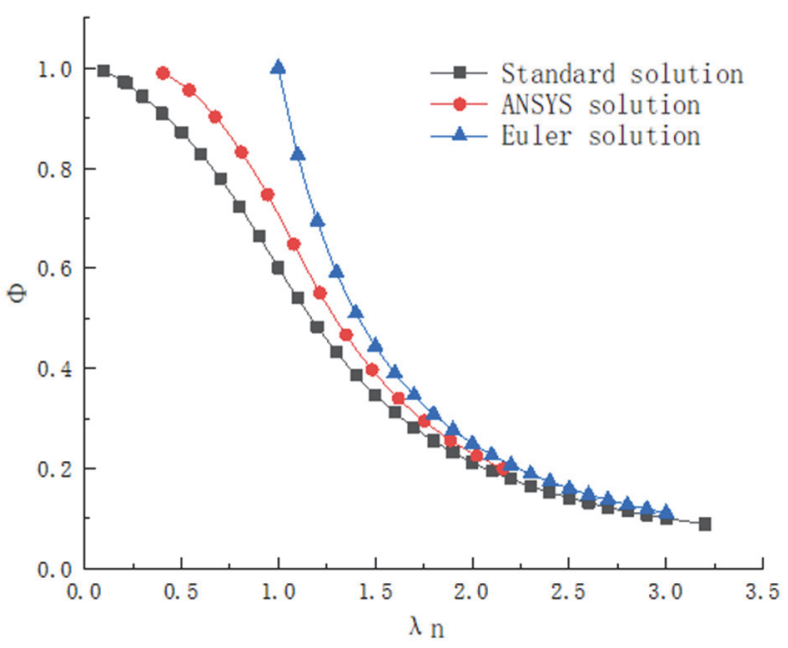

Figure 2Column curve under the initial bending of $L / 1,000$

The column curves above, which comprehensively consider the initial defects of geometry and materials, can be fitted into the standard expression below:

$\varphi=\frac{1}{2 \lambda_{n}^{2}}\left[\left(b_{1}+b_{2} \lambda_{n}+\lambda_{n}^{2}\right)-\sqrt{\left(b_{1}+b_{2} \lambda_{n}+\lambda_{n}^{2}\right)^{2}-4 \lambda_{n}^{2}}\right]$

where, the values of $b_{1}$ and $b_{2}$ are given in Tab. 2 .

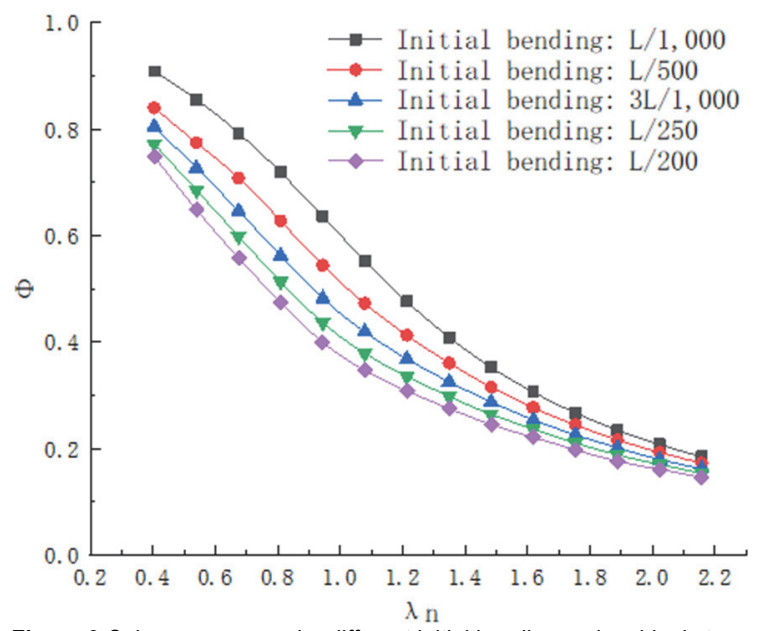

Figure 3 Column curves under different initial bending and residual stresses

Table 2 Values of $b_{1}$ and $b_{2}$

\begin{tabular}{|c|c|c|c|c|c|c|c|c|c|c|}
\hline & \multicolumn{2}{|c|}{$\mathrm{L} / 1,000$} & \multicolumn{2}{c|}{$\mathrm{L} / 500$} & \multicolumn{2}{c|}{$3 \mathrm{~L} / 1,000$} & \multicolumn{2}{c|}{$\mathrm{L} / 250$} & \multicolumn{2}{c|}{$\mathrm{L} / 200$} \\
\hline Parameter & $b_{1}$ & $b_{1}$ & $b_{1}$ & $b_{2}$ & $b_{1}$ & $b_{2}$ & $b_{1}$ & $b_{2}$ & $b_{1}$ & $b_{2}$ \\
\hline Value & 0.965 & 0.300 & 0.9688 & 0.4767 & 0.9306 & 0.6896 & 0.8946 & 0.8959 & 0.8465 & 1.115 \\
\hline$R$-squared & \multicolumn{2}{|c|}{1} & \multicolumn{2}{|c|}{0.9998} & \multicolumn{2}{c|}{0.9994} & \multicolumn{2}{c|}{0.9989} & 0.9984 \\
\hline
\end{tabular}

Through second-order elastic analysis, the $\varphi-\lambda_{n}$ of the axially compressed rods can be calculated by the edge yield criterion:

$\varphi=\frac{1}{2 \lambda_{n}^{2}}\left\{\left(1+\varepsilon_{0}\right)+\lambda_{n}^{2}-\sqrt{\left(\left(1+\varepsilon_{0}\right)+\lambda_{n}^{2}\right)^{2}-4 \lambda_{n}^{2}}\right\}$

where, $\varepsilon_{0}$ is the equivalent initial eccentricity:

$\varepsilon_{0}=\frac{A e_{0}}{W}$

$e_{0}$ is the midspan deflection with initial bending; $A$ is the cross-sectional area; $W$ is the cross-sectional modulus. Compared with Eq. (4), $\varepsilon_{0}$ can be described with $b_{1}$ and $b_{2}$ :

$\varepsilon_{0}=b_{1}+b_{2} \lambda_{n}-1$

Since $b_{1}$ and $b_{2}$ consider both geometric and material defects of rod pieces, the above expression of $\varepsilon_{0}$ describes the initial defects of components with geometric defects. Hence, Eq. (7) can be simplified to obtain the reference value of the initial defects of components $e_{0} / l$ :

$\frac{e_{0}}{l}=\frac{i}{y \lambda}\left(b_{1}+b_{2} \frac{\lambda}{\pi} \sqrt{\frac{f_{y}}{E}}-1\right)$

where, $\lambda$ is the slenderness ratio; $i$ is the turning radius; $f_{y}$ is the yield strength; $E$ is the elastic modulus. The relationship between $e_{0} / l$ and $\lambda$ can be derived by Eq. (8). Since $\lambda_{n}>0,215$ and the maximum allowable slenderness ratio of steel tubes for scaffold support is 250 (JGJ1302011) [15], the slenderness ratio $\lambda$ must fall within $11.63<$ $\lambda \leq 250$. Fig. 4 presents the $\frac{e_{0}}{l}-\lambda$ curves under different initial bending, with $11.63<\lambda \leq 250$. The maximum $e_{0} / l$ was taken as the reference value for equivalent initial defects (Tab. 3).

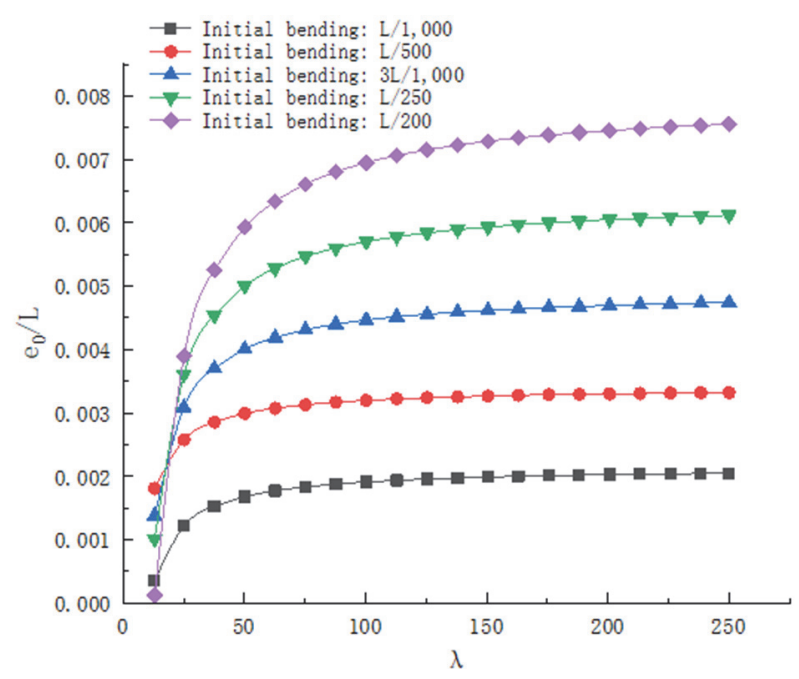

Figure $4 \frac{e_{0}}{l}-\lambda$ curves under different initial bending 
JGJ130-2011 defines the initial bending of steel tubes as $3 \mathrm{~L} / 1,000$ [15]. Under normal conditions, DAM design is recommended to adopt $\mathrm{L} / 210$ as the initial defects of components. If the steel tubes have been reused frequently, the initial bending should be measured on site; then, their initial defects could be determined accurately, according to the reference values in Tab. 3 .

Table 3 Reference values of initial defects of components at different initial bending

\begin{tabular}{|c|c|c|c|c|}
\hline Initial bending & $\mathrm{L} / 1,000$ & $\mathrm{~L} / 500$ & $3 \mathrm{~L} / 1,000$ & $\mathrm{~L} / 250$ \\
\hline Reference value of $e_{0} / l$ & $1 / 500$ & $1 / 300$ & $1 / 210$ & $1 / 160$ \\
\hline
\end{tabular}

\section{OVERALL INITIAL DEFECT OF STRUCTURE}

In JGJ130-2011, there is a whole section on scaffold checking and acceptance. That section reads: the allowable deviation of the verticality of vertical rods in high density scaffold is 3/1,000 of the rod height [15]. Drawing on GB 50017-2017, the section provides three ways to simulate the overall initial defect: (1) Apply an imaginary horizontal force on top of each layer of vertical rods in the direction of the most adverse reference load; (2) Apply an imaginary horizontal displacement on top of each layer of vertical rods in the direction of the most adverse reference load; (3) Adopt the consistent imperfect buckling analysis method with the lowest-order overall buckling mode and the maximum defect of $H / 250$ ( $H$ is structural height) [16]. Under external force, the STSC will move sideward. Thus, the third method leads to the smallest structural potential with overall initial defect and calculates the minimum bearing capacity as the ultimate bearing capacity.

In actual engineering, the geometric defects of the actual structure do not obey the distribution of the lowestorder overall buckling mode. Besides, the STSC is very sensitive to the overall initial defect. As a result, the third method often brings a far smaller outcome than the test results [9]. To solve the problem, the authors decided to apply the overall initial defect in the direction of the most adverse load, take the maximum deviation as $3 / 1,000$ of the structural height and adjust the geometric defects in other positions by the maximum deviation.

\section{EXAMPLE ANALYSIS \\ 4.1 Example Introduction}

The above quantification of the overall initial defect, and the initial defects of components lays a solid basis for the engineering application of the DAM. This paper illustrates the DAM application in STSC with a classic example from the full-scale test on a supertall high-density STSC by Liu et al. [17] and Lu [18] from Tianjin University. For such an STSC, the initial defects and semi-stiffness have a major impact on ultimate bearing capacity. Therefore, there is a huge difference in the results between the traditional analysis methods (e.g., linear buckling analysis and nonlinear analysis) and the DAM simulation. With this example, the authors demonstrated the flow and accuracy of the DAM, highlighting the key steps of the DAM. Tab. 4 presents the parameters of the example.

Table 4 Parameters of the example

Table 4 Parameters of the example
\begin{tabular}{|c|c|}
\hline Parameter & Value \\
\hline Horizontal distance $/ \mathrm{m}$ & $0.6 \times 5$ \\
\hline Longitudinal distance $/ \mathrm{m}$ & $0.6 \times 5$ \\
\hline Step length/m & $0.9 \times 8$ \\
\hline Extension height of vertical $\mathrm{rod} / \mathrm{m}$ & 0.5 \\
\hline Height of bottom horizontal tube $/ \mathrm{m}$ & 0.4 \\
\hline Tightening torque of coupler/ $\mathrm{N} \cdot \mathrm{m}$ & 40 \\
\hline
\end{tabular}

The cross-sectional parameters of the steel tubes are the same as in Tab. 1.

\subsection{Linear Buckling Analysis}

The linear buckling analysis was carried out on ANSYS. The rod pieces were simulated as BEAM188 elements. The nonlinear semi-stiffness of the nodes of horizontal and vertical rods was simulated by COMBIN39 spring elements. In this way, a finite-element model was established as shown in Fig. 5 (the defect application is detailed in the subsequent subsection). The structural load was designed as follows: 1.2 constant load +1.4 live load. The analysis shows that the ultimate bearing capacity of the STSC was $37.25 \mathrm{kN}$. Tab. 5 lists the buckling eigenvalues in the first six orders. Fig. 6 shows the lowest-order overall buckling mode. It can be seen that the entire scaffold bulged significantly and became instable.

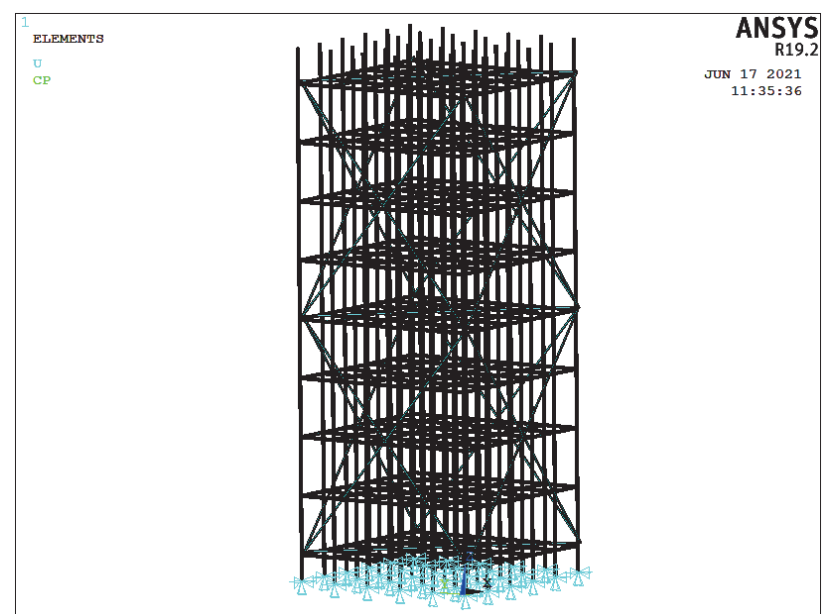

Figure 5 Finite-element model of the supertall high-density STSC

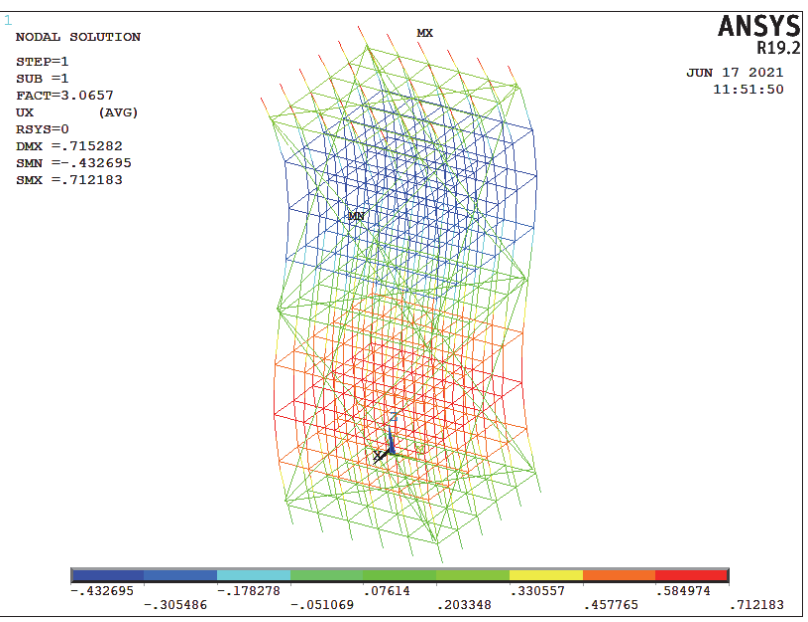

Figure 6 Lowest-order overall buckling mode 
The buckling factor $\eta_{\text {cr }}$ equals the first-order buckling eigenvalue of 3.0657. Thus, the second-order effect coefficient $\theta_{i}^{\text {II }}$ can be calculated by:

$$
\theta_{i}^{\mathrm{II}}=\frac{1}{\eta_{c r}}
$$

Table 5 Buckling eigenvalues in the first six orders

\begin{tabular}{|c|c|}
\hline Order & Eigenvalue \\
\hline 1 & 3.0657 \\
\hline 2 & 3.0662 \\
\hline 3 & 4.1171 \\
\hline 4 & 4.1331 \\
\hline 6 & 5.1971 \\
\hline
\end{tabular}

It is calculated that the $\theta_{i}^{\mathrm{II}}=0.326>0.1$. According to GB 50017-2017, linear buckling analysis is not suitable; the calculated ultimate bearing capacity deviated far from the measured value of $32.5 \mathrm{kN}$. Therefore, DAM is necessary for analyzing the calculation example.

\subsection{DAM Analysis}

The key of the DAM analysis is to perform a nonlinear analysis based on the initial defects of structure and components, the semi-stiffness of nodes, and the nonlinearity of materials; the analysis must consider the effects of $P-\Delta$ and $P-\delta$. Therefore, DAM raises a high requirement on the fineness of modeling. Specifically, the overall initial defect was designed as the initial displacement with $3 / 1,000$ of the structural height as the maximum deviation, i.e., $\Delta=8.1 \mathrm{~m} \times 3 / 1,000=0.0243 \mathrm{~m}$; the maximum initial displacement occurred on the top of the scaffold; the coordinates of other nodes were linearly adjusted to reflect the $P-\Delta$ effect of the structure. The initial defects of rod pieces were simulated by applying an initial displacement of L/210 of the rod length. Each rod piece was divided into 4 segments. The node coordinates were adjusted by the sinusoidal curve to consider the $P-\delta$ effect of the components. In addition, the node semistiffness has a non negligible impact on stable bearing capacity. Hence, the semi-stiffness of the nodes of horizontal and vertical rods was simulated as COMBIN39 nonlinear spring elements. The nonlinear rotational stiffness was defined by real constant inputs of $M-\theta$ data, which come from the test data provided by China State Construction Technical Center (Fig. 7). Based on these data, the authors established a refined model considering $P$ $-\Delta$ effect, $P-\delta$ effect, and node semi-stiffness.

Considering the geometric and material nonlinearity of the structure, the nonlinearity of the structure was considered as the ideal elastoplastic city, and the arc length method was adopted for nonlinear calculation. In this way, the structural displacement under ultimate bearing capacity (Fig. 8) and the load-displacement curve of the maximum displacement point (along the application direction of overall defect) (Fig. 9). It can be seen that the ultimate bearing capacity of the high-density scaffold was $31.98 \mathrm{kN}$, only $1.6 \%$ smaller than the test result of $32.5 \mathrm{kN}$ [17]; the maximum node displacement was $42.6 \mathrm{~mm}$; the instability exhibited as the top of the vertical rod extending out, causing the scaffold to move sideward, and the bottom rod pieces were not significantly deformed. The instability mode was basically the same as the test observations.

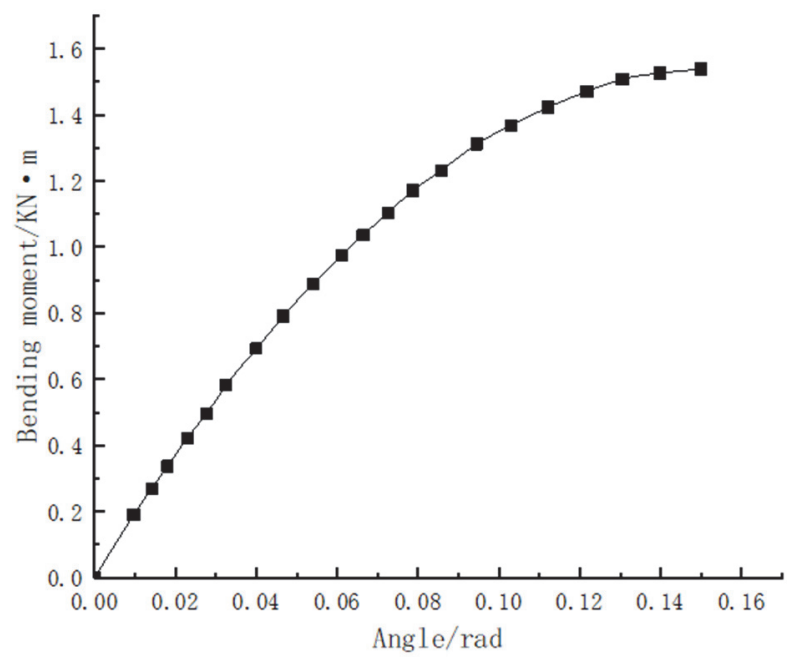

Figure 7 Bending moment-angle curve for node semi-stiffness simulation

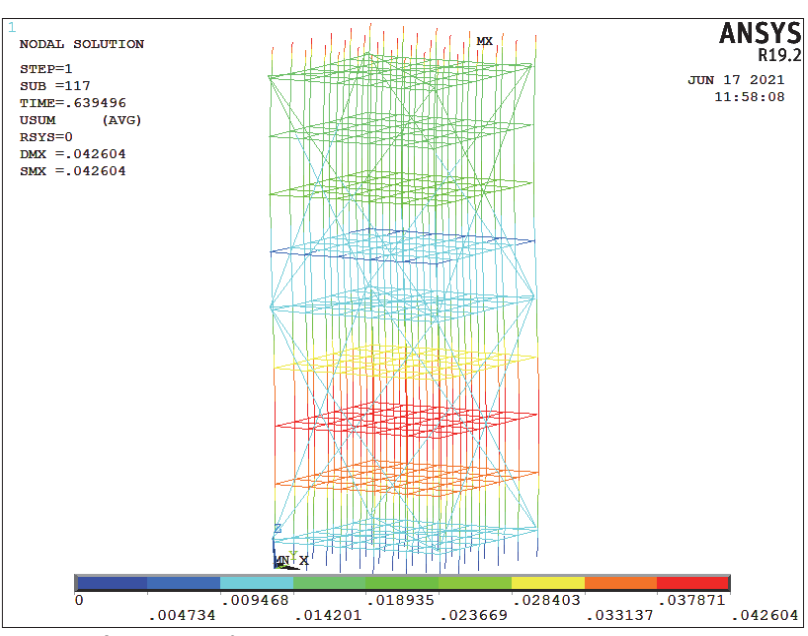

Figure 8 Cloud map of structural displacement under ultimate bearing capacity

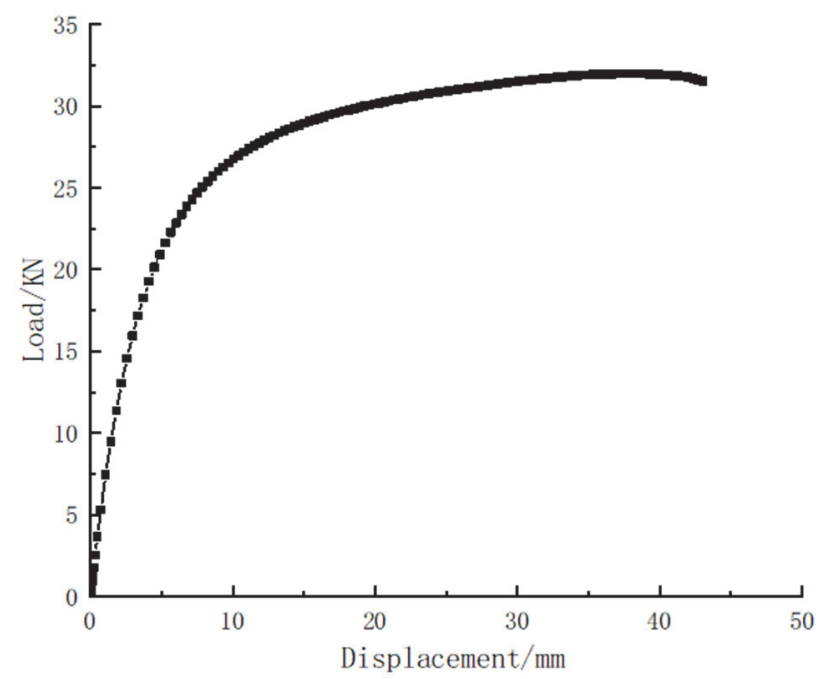

Figure 9 Load-displacement curve at the maximum displacement node

\section{COMPARATIVE ANALYSIS OF RESULTS}

The overall defect of the example was applied directly in the modeling process. Since the scaffold is highly sensitive to structural displacement, the ultimate bearing 
capacity obtained after the overall initial defect was applied under the lowest-order overall buckling mode declined sharply. Fig. 10 compares the ultimate bearing capacities calculated by linear buckling analysis, DAM analysis, lowest-order overall buckling mode analysis, and JGJ1302011. The following conclusions can be drawn:

(1) The ultimate bearing capacity obtained by linear buckling analysis was $37.25 \mathrm{kN}, 14.6 \%$ away from the test result; the ultimate bearing capacity obtained by DAM analysis was $31.98 \mathrm{kN},-1.6 \%$ away from the test result; the ultimate bearing capacity obtained by the lowest-order overall buckling mode analysis was $25.46 \mathrm{kN},-21.7 \%$ away from the test result. Overall, the DAM analysis achieved the smallest error and the best results among the three methods. This means the DAM can accurately reflect the internal force response of the structure, and the reference values of overall initial defects are reasonable.

(2) The stable bearing capacity of the whole structure declined sharply after the initial defects were applied under the lowest-order overall buckling mode, indicating that the high-density scaffold is highly sensitive to displacement defect. It is suggested to simulate the overall initial defect of the scaffold through DAM simulation.

(3) The allowable bearing capacity obtained by JGJ130-2011 was $13.39 \mathrm{kN}$. The safety factors between this value and the results of the test and DAM were no smaller than 2.0. Thus, the calculation result can be applied to real projects.

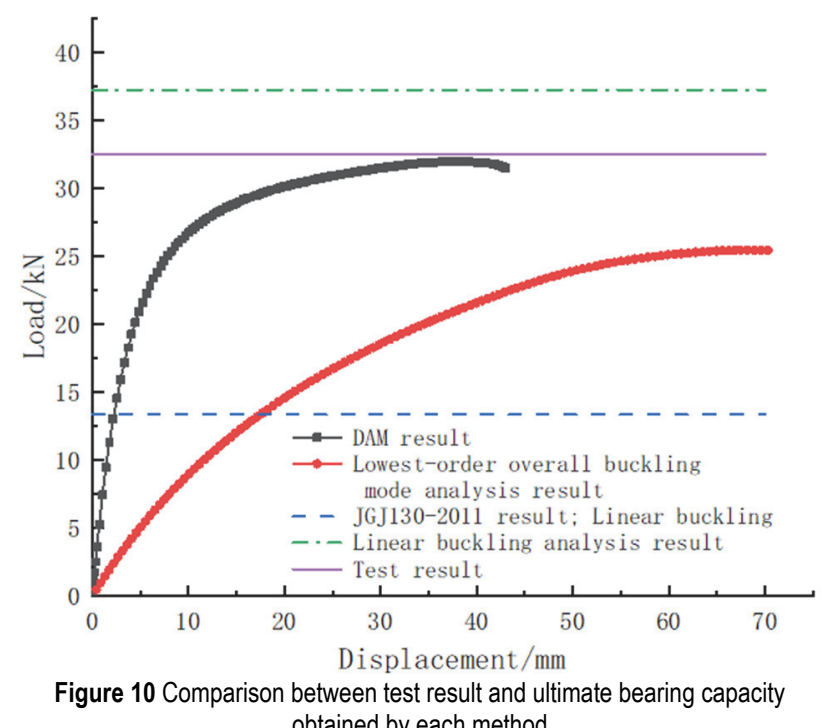

\section{CONCLUSIONS}

(1) For frequently reused rod pieces, it is recommended to measure the initial bending on the site, and consider the $P-\delta$ effect of each rod, according to the proposed reference values for overall initial defects.

(2) The application of the DAM in STSC raises a high requirement on the fineness of modeling. To ensure the analysis accuracy, it is recommended to consider the $P-$ $\Delta$ effect with a displacement about $3 / 1,000$ of structural height in the most adverse load direction, or determine the overall initial defect through preassembling on site; reflect the $P-\delta$ effect by dividing each rod into several segments (at least 4 to ensure accuracy), with a displacement about
$1 / 210$ of rod length; describe the node semi-stiffness with the bending moment and angle measured by test, as well as the nonlinear rotational stiffness of the node. In addition, if the structure rests on an existing structure or a soft and weak foundation, it is also necessary to consider the influence of boundary conditions on the stabile carrying capacity of the structure.

(3) DAM-based analysis of the STSC focuses on overall stability. The analysis results are accurate and reliable. This analysis strategy only needs to verify the cross-sectional strength of rod pieces, eliminating the need for additional calculation of the rod stability. Thus, this strategy provides more accurate quantified results to STSC analyses and designers. It is suggested doubling the safety factor of the analysis results for use in actual projects.

\section{Acknowledgements}

This work is supported by National Key Research and Development (R\&D) Plan, China (2018YFCO808405), and 2018 Science and Technology Plan, Xi'an Municipal Science and Technology Bureau, China (201844).

\section{REFERENCES}

[1] Chen, W. F. \& Lui, E. M. (1987). Structural stability: Theory and implementation. New York: Elsevier.

[2] Wang, M., Wang, L. J., Yu, H. Q. Tan, J. P., \& Lu, L. J. (2016). Application of direct analysis method in spatial structure design. Construction Technology Editorial Department, 45, 373-378

[3] Liu, Z. K., Jin, L. J., Zhou, X. H., \& Ma, Y. W. (2021). Stateof-the-art on research of direct analysis method of steel members with global instability. Journal of Building Structures, 42(8), 1-12.

[4] Yu, Z., Xue, S. Z., \& Wang, L. J. (2021). Application of direct analysis design method in cable structure design. Building Structure, 51(1), 45-49.

[5] Zhou, L. (2021). Structural stability analysis of steel roof of Yanghang Sports Center based on direct analysis method. Journal of Building Structures, 50(S2), 580-584.

[6] Hu, C. M. \& Zeng, F. K. (2010). A study of experiment and numerical model on coupler steel tube falsework. Engineering Mechanics, 27(Suppl.II), 280-283, 293.

[7] Lu, Z. R.,Chen, Z. H., Wang, X. D., Liu, Q., \& Liu, H. B. (2012). Experimental and theoretical study of the bearing capacity of fastener steel tube full-hall formwork support system. China Civil Engineering Journal, 45(1), 49-60.

[8] Yuan, X. X., Jin, W. L., Lu, Z., Liu, X., \& Chen, T. M. (2006). A study on the stability bearing capacity of fastenerstyle tubular steel formwork-supports. China Civil Engineering Journal, 39(5), 43-50. https//doi.org/10.15951/j.tmgcxb,2006. 05. 007

[9] Yao, X. (2014). Study on calculation method of initial defects of tall formwork support with coupler. Chongqing University.

[10] Liu, W. \& Shu, G. P. (2021). Study on equivalent initial defects of compression component in direct analysis and design method.Journal of Building Structures, 42(6), 199205. https://doi.org/10.3390/buildings11050199

[11] Zhang, W. H., Liu, J. M., \& Zhu, G. W. (2009). A semi-rigid node calculation method for steel tubular scaffold with complete based on back-analysis of scaffold load-bearing experiments results. Journal of Shandong jianzhuuniversity, 24(1), 38-43.

[12] Prabhakaran, U., Godley, M. H. R., \& Beale, R. G. (2006). Three-dimensional second order analysis of scaffolds with 
semi-rigid connections. In Tubular Structures XI: 11th International Symposium and IIW International Conference on Tubular Structures, 11, 203. https://doi.org/10.1201/9780203734964-25

[13] Hu, C. M., Che, J. L., Zhang, H. Z., \& Dong, P. (2010). Influence of semi-rigid joint on stability capacity of coupler steel tube falsework.Industrial Construction, 40(2), 20-23. https//doi.org/10.13204/j.gyjz201002005

[14] Zou, A. M., Li, Q. W., He, M. H., \& Zhang, H. (2016).FEA on bearing behavior of cuplok scaffold based on tri-linear semi-rigid joint model. Journal of Building Structures, 37(4), 151-157.

[15] JGJ 130-2011, Technical Code for Safety of Steel Pipe Scaffold with Coupler in Construction.Beijing: China Building Industry Press.

[16] GB 50017-2017, Code for Design of Steel Structures. Beijing: China Building and Building Press.

[17] Liu, H. B., Chen, Z. H., Wang, X. D., \& Liu, Q. (2011). Simplified Calculation Method of Steel Tube and Coupler Scaffold with X-Bracing. Journal of Civil, Architectural \& Environmental Engineering, 33(4), 65-72, 79. https//doi.org/10.11835/j.issn.1674-4764.2011.04.011

[18] Lu, Z. R. (2010). Theoretical analysis and experimental research on coupler type full steel tube supporting system. Tianjin University. https//doi.org/10.7666/d.y1925875

\section{Contact information}

\section{Junce ZHANG}

School of Civil Engineering and architecture

Xi'an Technological University,

Xi'an 710021, China

E-mail: zhangjc1003@163.com

\section{Fankui ZENG}

(Corresponding author)

School of Civil Engineering and architecture

Xi'an Technological University,

Xi'an 710021, China

E-mail: fankuizeng@126.com

\section{Huan MA}

School of Civil Engineering and architecture,

Xi'an Technological University,

Xi'an 710021, China

E-mail: Mahuan13991488110@163.com 